Cell Research(1994),4, 163-172

\title{
Observation of nuclei reassembled from demembranated Xenopus sperm nuclei and analysis of their lamina com- ponents
}

\author{
QU J J IAN $, \mathrm{C}_{\text {HUAn ma o }} \mathrm{ZHANG}, \mathrm{Z}_{\text {Honghe }} \mathrm{ZHAI}$. \\ College of Life Sciences, Beijing University, Beijing \\ 100871, China.
}

\begin{abstract}
A cell-free preparation obtained from extracts of activated Xenopus laevis eggs induced chromatin decondensation and nuclear formation from demembranated Xenopus sperm nuclei. Electron microscopy revealed that the reassembled nucleus had a double-layered nuclear membrane, nuclear pore complexes, and decondensed chromatin etc. Indirect immunofluorescence analysis demonstrated the presence of lamina in newly assembled nuclei. Western-blotting results showed that lamin $\mathrm{L}_{I I}$ was present in egg extracts and in lamina of the reassembled nuclei which were previously reported to contain only egg derived lamin $\mathrm{L}_{I I I}$.
\end{abstract}

Key words: Nuclear assembly in vitro, Xenopus laevis, Lamina.

\section{INTRODUCTION}

Following the pioneer work of Lokha and Masui[1, 2], studies using cell-free system have greatly enriched our knowledge about the events during cell cycle. Extracts derived from activated Xenopus laevis eggs able to asemble nuclei around exogeneous naked DNA, isolated chromosomes and demembranated sperm chromatin have been widely used to study the pathways and biochemistry of nuclear envelope and pore complexes assembly, lamina formation, DNA replication and nuclear cytoplasmic transportation[3-6]. Meanwhile, extracts prepared from mataphase eggs were adopted to study spindle organization and nuclear disassembly[7-10]. Plenty of information on cell cycle regulation concerning nuclear reassembly and mitosis has also been obtained from the studies using cell-free system[9, 10]. Since 1989, colleagues 
Nuclei assembled in vitro and their lamina components

in our laboratory have devoted to this field and achieved a number of results[11-14]. Recently we have successfully got the nuclei assembled in vitro around demembranated sperm chromatin in activated Xenopus egg extracts. The nuclei assembled in our system were morphologically similar to those of intact cells.

The nuclear lamina, a fibrous network located between nuclear envelope and chromatin, is composed of lamins. It has long been proposed that lamin proteins play important roles in nuclear assembly, chromatin topology and DNA replication and the interactions among membrane, lamins and chromatin bring about these processes[1518]. Molecular and biochemical anaylyses have revealed that the lamin proteins are highly conserved and developmentally expressed in a tissue-specific manner. Based on comparison of their primary structure, biochemical features and expression pattern, two major lamin isotypes can be distinguished, A-type lamins (mammalian laminA and C, chicken and Xenopus lamin A) and B-type lamins which seem to be constitutively expressed and can be further classified into B1 (mammalian and chicken lamin B1 and Xenopus lamin $\mathrm{L}_{\mathrm{I}}$ ), B2 (mammalian and chicken B2 and Xenopus lamin $\mathrm{L}_{I I}$ ) and B3 Xenopus lamin $\mathrm{L}_{I I I}$ ) type lamins. When the nuclear envelope breaks down during mitotic or meiotic cell division, lamin filaments are hyperphosphorylated by the protein kinase P34 ${ }^{\mathrm{cdc} 2}$ and depolymerized into oligomers. While A-type lamins are dispersed throughout the cytoplasm as soluble oligomers being not in contact with membranes, B-type lamins, though depolymerized, remain associated with the reminants of the nuclear envelope[19-22].

Lamin $\mathrm{L}_{I I}$ is reported to be the single lamin isoform in Xenopus oocytes, eggs and early embryos and is present in embryos up to the tail bud stages, reappearing only in certain adult cells (neurons, muscle cells, and diplotene oocytes)[21]. Though classified as B-type lamin it is soluble during nuclear envelope break-down[19-21]. Lamin $\mathrm{L}_{I}$ and $\mathrm{L}_{I I}$ appear at characteristic stages in development. Lamin $\mathrm{L}_{I}$ first appears at midblastula transition and $\mathrm{L}_{I I}$ at the gastrula [21]. Considering the importance of lamin proteins in normal cells, we were prompted to examine whether the nuclei assemnled in vitro had lamina structure and what the lamin components were. Our results showed that the reassembled nuclei did have lamina and, interestingly, lamin $\mathrm{L}_{I I}$ was found to be present in egg extracts and in nuclei assembled in vitro.

\section{MATERIALS AND METHODS}

\section{Preparation of sperm nuclei}

Sperm nuclei were prepared as described by Lohka and Masui[1] with some modifications. Testes were dissected from sexually mature $X$. laevis which had been injected with 100 I.U. human chorionic gonadotropin (HCG) and kept for $1 \mathrm{~h}$ at $22^{\circ} \mathrm{C}$. The testes were washed free of blood and incubated overnight at $18^{\circ} \mathrm{C}$ in $200 \%$ Steinberg's solution containing antibiotics and HCG(10 I.U./ml). Sperms were released by gently squeezing the testes, harvested by centrifugation at $1,500 \mathrm{~g}$ for 10 min and washed 3 times with nuclear isolation buffer (NIB, $15 \mathrm{mmol} / \mathrm{L} \mathrm{NaCl}, 60 \mathrm{mmol} / \mathrm{L} \mathrm{KCl}, 15$ $\mathrm{mmol} / \mathrm{L}$ Tris, $\mathrm{pH}$ 7.4, $1 \mathrm{mmol} / \mathrm{L} \mathrm{DTT}, 0.5 \mathrm{mmol} / \mathrm{L}$ spermine, $0.15 \mathrm{mmol} / \mathrm{L}$ spermidine). Sperms 
were treated with NIB containing lysolecithin at $330 \mu \mathrm{g} / \mathrm{ml}$ and proteinase inhibitor for $10 \mathrm{~min}$ at $22{ }^{\circ} \mathrm{C}$. Lysolecithin -treated sperms were washed once with ice-cold NIB/3\% bovine serum albumin (BSA), 3 times with NIB/0.4\% BSA and finally resuspended in NIB/30\% glycerol. They were counted with a hemocytometer, and stored at $-70^{\circ} \mathrm{C}$.

\section{Preparation of HeLa cell chromosome clusters}

As previously described[14], HeLa cells were cultured in E-MEM (Eagle's modified essential medium) and synchronized at metaphase by treatment with TdR and nocodazole. Cells were swelled in 1/2 Hank's solution for $2 \mathrm{~h}$ and then homogenized. Chromosome clusters were pelleted by centrifugation at $1,000 \mathrm{~g}$ for $10 \mathrm{~min}$, resuspended in E-MEM containing $20 \%$ newborn calf serum and $10 \%$ glycerol and stored at $-70^{\circ} \mathrm{C}$.

\section{Preparation of egg extracts}

Egg extracts were prepared as previously described[11]. Briefly, unfertilized eggs were collected from HCG-treated mature female $X$. laevis and dejellied with $2 \%$ cysteine. After activated by $\mathrm{Ca}^{2+}$ ionophore A23187, they were washed with buffer containing cycloheximide and cytochalasin B. Then the eggs were crushed at $10,000 \mathrm{~g}, 4^{\circ} \mathrm{C}$ for $15 \mathrm{~min}$. The material between the lipid top layer and yolk pellet was removed and centrifuged again at $10,000 \mathrm{~g}, 4^{\circ} \mathrm{C}$ for $15 \mathrm{~min}$. The supernatant was used as egg extracts.

\section{Nuclear assembly in vitro}

Demembranated sperm nuclei and clusters of HeLa cell chromosomes were respectively mixed with freshly prepared egg extracts at a concentration of $10^{3}$ nuclei or chromosome clusters/ $\mu$ l egg extracts and then the ATP regenerating system $(2 \mathrm{mmol} / \mathrm{L}$ ATP, $50 \mu \mathrm{g} / \mathrm{ml}$ creatine kinase, $2 \mathrm{mmol} / \mathrm{L}$ phosphocreatine) was added. The mixture was incubated at $22^{\circ} \mathrm{C}$.

\section{Antibodies}

All antibodies used here were antilamin monoclonal antibodies generously donated by Dr. Krohne. They were antilamin $\mathrm{L}_{I I} / \mathrm{L}_{I I I} \mathrm{mAb} \mathrm{X} 155$, antilamin $\mathrm{L}_{I I} \mathrm{mAb} \mathrm{X} 223$ and antilamin $\mathrm{L}_{I} / \mathrm{L}_{I I} /$ lamin A mAb X167[26].

\section{Immunofluorescent microscopy}

Incubated mixtures were smeared onto glass slide, air-dried for 5 min and fixed for $30 \mathrm{~min}$ in mixture of acetone and methanol $(1: 1)$ at $-20^{\circ} \mathrm{C}$. After incubated in PBS containing $1 \%$ BSA for $10 \mathrm{~min}$, the specimen were incubated with mAb X155 for $1 \mathrm{~h}$, and washed 3 times with PBS. Then FITC labeled goat anti-mouse IgG was added and the specimen were incubated for $30 \mathrm{~min}$ and washed 3 times with PBS. Chromatin was stained by DAPI (DNA dye). The specimen were mounted with a coverslip and examined by fluorescence microscope.

\section{Electron microscopy}

Aliquots of the incubation mixture were fixed in ice-cold $2 \%$ glutaraldehyde and osmium tetroxide successively, dehydrated through a graded concentration series of acetone and then embedded in Epon 812. Ultrathin sections were cut, stainned with uranyl acetate and lead citrate, and finally examined under JEM-100CX transmission electron microscope.

\section{Gel electrophoresis and immunoblotting}

After diluted 10 times with NIB, the incubation mixture was centrifuged at 3,000g for $10 \mathrm{~min}$. The pelleted nuclei were washed 3 times with NIB and then suspended in SDS sample buffer. Proteins were resolved on 10\% SDS-PAGE and transferred to nitrocellulose filters. Filters were blocked 
Nuclei assembled in vitro and their lamina components

$\left(37^{\circ} \mathrm{C}\right)$ in $3 \% \mathrm{BSA} / \mathrm{TBS}(200 \mathrm{mmol} / \mathrm{L}$ Tris, $500 \mathrm{mmol} / \mathrm{L} \mathrm{NaCl}, \mathrm{pH} 7.5)$ for $2 \mathrm{~h}$ and then incubated $\left(37^{\circ} \mathrm{C}\right)$ in $1 \% \mathrm{BSA} / \mathrm{TTBS}(200 \mathrm{mmol} / \mathrm{L}$ Tris, $500 \mathrm{mmol} / \mathrm{L} \mathrm{NaCl}, \mathrm{pH} 7.5,0.05 \%$ Tween 20$)$ containing antilamin antibody mAb X155 or mAb X223 or mAb X167 for $3.5 \mathrm{~h}$. After washed with TTBS and incubated in 1\% BSA /TTBS containing Biotin labeled goat anti-mouse IgG for 2 $\mathrm{h}$, filters were washed with TTBS and then blocked in 3\% BSA/TBS for $1.5 \mathrm{~h}$. Filters were incubated in buffer 1 (100 mmol/L Tris, $1 \mathrm{~mol} / \mathrm{L} \mathrm{NaCl}, 2 \mathrm{mmol} / \mathrm{L}_{\mathrm{MgCl}_{2},}, 0.05 \%$ TritonX-100, $\mathrm{pH} 7.5$ ) containing Avidin-AP (akaline phosphotase) for $15 \mathrm{~min}$ and then washed successively with buffer 1 and buffer $2\left(100 \mathrm{mmol} / \mathrm{L}\right.$ Tris, $1 \mathrm{~mol} / \mathrm{L} \mathrm{NaCl}, 5 \mathrm{mmol} / \mathrm{L} \mathrm{MgCl}_{2}, \mathrm{pH}$ 9.5). Filters were stained in buffer 3 (100 mmol/L Tris, $100 \mathrm{mmol} / \mathrm{L} \mathrm{NaCl}, 5 \mathrm{mmol} / \mathrm{L} \mathrm{MgCl}_{2}$, $\mathrm{pH}$ 9.5) containing NBT and BCIP.

\section{RESULTS AND DISSCUSSION}

Incubation of demembranated Xenopus sperm nuclei in egg extracts resulted in rapid chromatin decondensation followed by nuclear formation. The demembranated sperm chromatin was in a long, thin and condensed form which was the characteristic of the sperm nucleus and could be stained strongly with DAPI[Fig 1]. When it was added to egg extracts and incubated at certain temperature $\left(22^{\circ} \mathrm{C}\right)$, the sperm chromatin began to elongate and swell, showing discernible decondensation, in the first $10 \mathrm{~min}$ of incubation[Fig 2]. After 60-90 min, nearly all of the originally sharpcontoured demembranated sperm nuclei had decondensed and changed into spherical shape like a pronucleus[Fig 3], while less than 5\% of them remained in their initial form. Most of the added sperm chromatin followed the same steps in assuming morphological alteration[Fig 4]. Lysolecithin was effective in removing cell membrane and nuclear envelope of sperm. At the ultrastructral level, we observed that after it was treated with lysolecithin, sperm chromatin was devoid of double-layered nuclear envelope and was rather condensed[Fig 5]; After incubation for about $1 \mathrm{~h}$, the decondensed chromatin gradually acquired a continuous nuclear envelope with pores. The newly assembled nuclei were morphologically indistinguishable from those in intact interphase cells[Fig 6].

Nuclei assembled in vitro were assessed for the presence of lamina by indirect immunofluorescent microscopy. When incubated with mAb X155 (specific for lamin $\left.\mathrm{L}_{I I} / \mathrm{L}_{I I I}\right)$, the demembranated sperm nuclei had no positive staining while the newly assembled nuclei exhibited positive rim-staining[Fig 7]. It was reported that only lamin $\mathrm{L}_{I V}$ was found in spermatids and spermatozoa[27]. Due to the lack of antibody specific for lamin $\mathrm{L}_{I V}$, we could not make sure whether the demembranated sperm nuclei still had some attached lamin $\mathrm{L}_{\text {Iv }}$ protein, although they had no intact lamina structure after demembranation. However, even though we could not conclude from the negative result that there was not any type of lamin protein around the periphery of demembranated sperm nuclei, we surely knew that there was no lamin $\mathrm{L}_{I I}$ or $\mathrm{L}_{I I I}$ protein; and then from the positive result we knew that during incubation, lamin $\mathrm{L}_{I I}$ or/and $\mathrm{L}_{I I I}$ which were previously stored in egg extracts participated in the formation of lamina structure of the newly assembled nuclei. Our previous work had demonstrated that nuclei assembled around clusters of HeLa cell chromosomes in egg extracts also had lamina [14]. 
$\mathrm{Qu} \mathrm{J}$ et al.
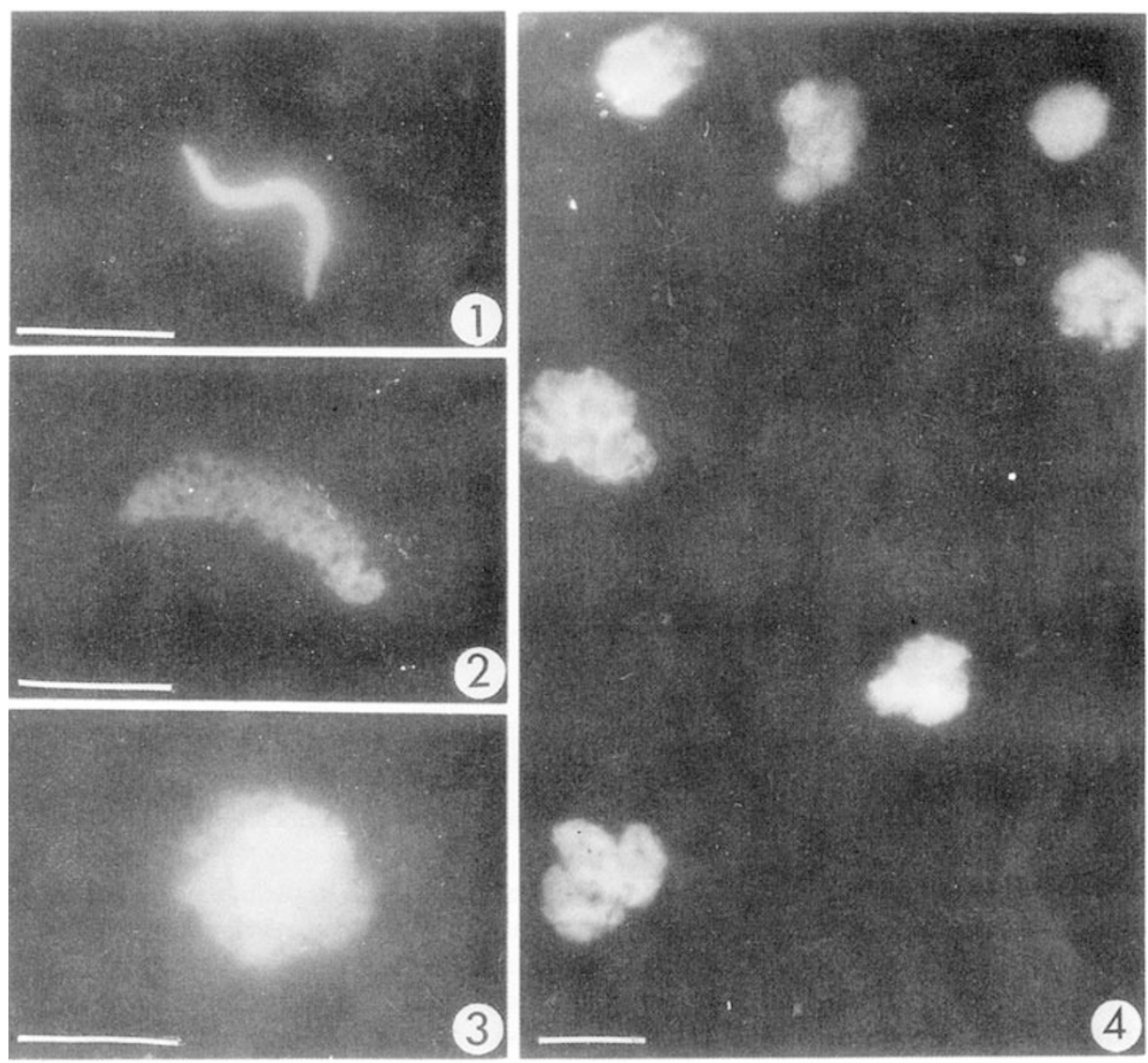

Fluorescence microscopy (DAPI stain) showing the decondensation of demembranated sperm nuclei in egg extracts. Bars: $10 \mu \mathrm{m}$.

Fig 1. The highly condensed sperm chromatin before incubation.

Fig 2. Elongated and swelled sperm chromatin after incubation of $10 \mathrm{~min}$.

Fig 3. Highly decondensed sperm chromatin taking a spherical shape after incubation of $60 \mathrm{~min}$.

Fig 4. Most of the added demembranated sperm nuclei followed the same steps to decondense to a spherical shape after incubation of $60 \mathrm{~min}$.

Western-blotting analysis was used to detect the lamina components of nuclei assembled in vitro. Nuclei were isolated from the incubation mixture by centrifugation and proteins were separated by gel-electrophoresis and then transferred to nitrocellulose filters. The fiters were probed with antilamin $\mathrm{L}_{I I} / \mathrm{L}_{I I I} \mathrm{mAb} \mathrm{X} 155$, antilamin $\mathrm{L}_{I I} \mathrm{mAb} \mathrm{X} 223$, or antilamin $\mathrm{L}_{I} / \mathrm{L}_{I I} /$ lamin $\mathrm{A} \mathrm{mAb} \mathrm{X} 167$ respectively. Using $\mathrm{mAb}$ $\mathrm{X} 155$ we found that both demembranated sperm nuclei and chromosomes of HeLa cells had no egg-derived lamin, whereas nuclei assembled in vitro from both of them 
Nuclei assembled in vitro and their lamina components

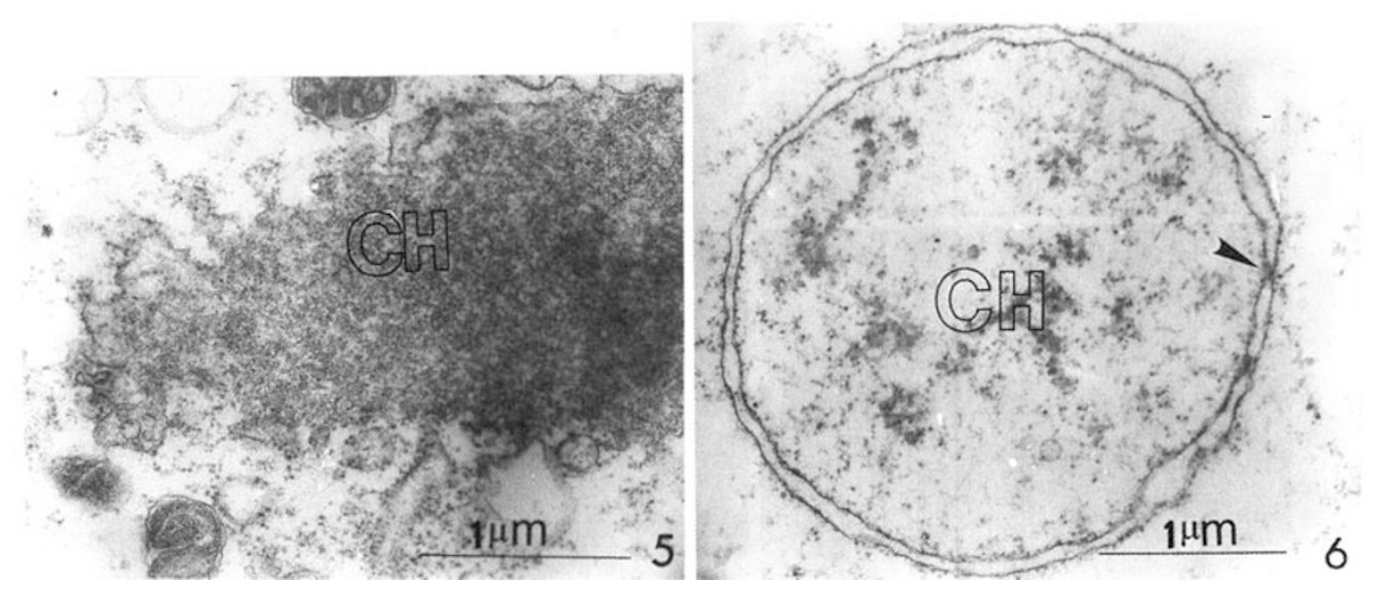

Ultrathin section of demembranated sperm chromatin and nuclei assembled in vitro. Bars: $1 \mu \mathrm{m}$

Fig 5. Demembranated sperm chromatin devoid of nuclear envelope.

Fig 6. Typical nuclei assembled in vitro with continuous nuclear envelope, $\operatorname{pores}(\uparrow)$ and well-decondensed chromatin.

contained the same lamin components as those found in egg extracts[Fig 8]. This confirmed our observation by indirect immunofluorescent microscopy that the lamins in the newly formed nuclei were derived from egg extracts. It is widely believed that $X$. laevis oocytes, eggs and early embryos contain only one lamin isoform, the lamin $\mathrm{L}_{I I I}$, which serves as a maternal pool for the formation of lamina in the pronuclei and early cleavage nuclei. During development lamin $\mathrm{L}_{I I I}$ is replaced in embryonic nuclei by lamin $\mathrm{L}_{I}$ and $\mathrm{L}_{I I}$ at the midblastula and gastrula stages respectively[21]. Considering that lamin $\mathrm{L}_{I I}$ and $\mathrm{L}_{I I I}$ had the same molecular weight of $68 \mathrm{kD}$ and $\mathrm{mAb} \mathrm{X} 155$ recognized both lamin $\mathrm{L}_{I I}$ and $\mathrm{L}_{I I I}$, we then used $\mathrm{mAb} \mathrm{X} 223$ and $\mathrm{mAb}$ $\mathrm{X} 167$ which both reacted with lamin $\mathrm{L}_{I I}$ but not with lamin $\mathrm{L}_{I I I}[26]$. To our interest, we found that both egg extracts and the nuclei assembled in the egg extracts reacted positively with the two antibodies. These results unequivocally suggested that besides lamin $\mathrm{L}_{I I I}$, lamin $\mathrm{L}_{I I}$ was also present in egg extracts and was involved in lamina formation of the nuclei assmbled in vitro in egg extacts[Fig 9, 10]. When we are preparing this paper, it comes to our notice that the recent work of Lourim and Krohne also confirmed the existence of lamin $\mathrm{L}_{I I}$ in Xenopus oocytes, eggs and embryos[26]. 
$\mathrm{Qu} \mathrm{J}$ et al.

Fig 7. Indirect immunofluorescent staining of lamina in the nucleus assembled in vitro with antilamin $\mathrm{mAb}$ X155. Bars: $10 \mu \mathrm{m}$.
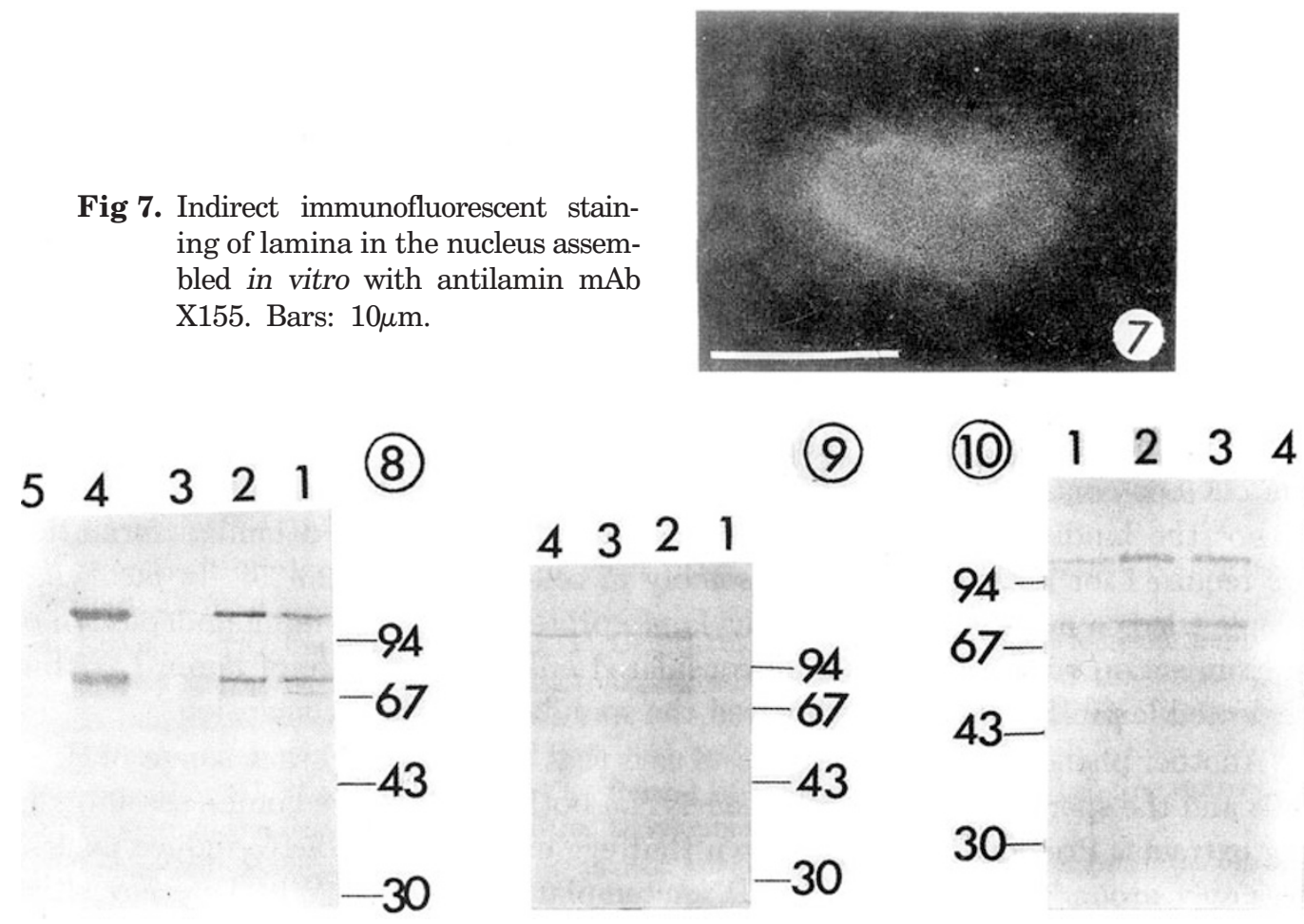

Fig 8. Both the nuclei assembled in vitro around sperm chromatin and chromosomes of HeLa cells had the same lamin peptides as those in egg extracts. Proteins were separated by SDS-PAGE and transferred to NC filters and then probed with mAb X155 in combination with ABC-Western-blotting technique. 1. egg extracts. 2.nuclei assembled around demembranated sperm nuclei. 3.demembranated sperm nuclei. 4. nuclei reassembled around chromosomes of $\mathrm{HeLa}$ cells. 5. chromosomes of HeLa cells. Molecular weight was showed at right in $\mathrm{kD}$.

Fig 9. Lamin $\mathrm{L}_{I I}$ was present in egg extracts and nuclei assembled in vitro detected by $\mathrm{mAb}$ X167. 1. egg extracts. 2.nuclei assembled around chromosomes of HeLa cells. 3. chromosomes of HeLa cells. 4.nuclei assembled around demembranated sperm nuclei.

Fig 10. Lamin $L_{I I}$ was present in egg extracts and nuclei assembled in vitro detected by mAb X223. 1. egg extracts. 2. nuclei assembled around demembranated sperm nuclei. 3. nuclei assembled around chromosomes of HeLa cells. 4. chromosomes of HeLa cells.

In discussing lamina formation of mammalian cells, Gerace and Blobel proposed a different functional specialization for lamin A and B in terms of chromatin and membrane binding respectively[22]. Since only lamin $\mathrm{L}_{I I I}$ is found in $X$. laevis oocytes, eggs and early embryos, it has been suggested that lamin $\mathrm{L}_{I I I}$ alone can build up a lamina structure in early cleavage nuclei and carry out both functions of chromatin-binding and membrane-binding in lamina formation through the mediation of some kinds of membrane or chromatin associated receptors. It has been 
Nuclei assembled in vitro and their lamina components

hypothesized that different lamins are functionally equivalent during the developmental change of lamina composition in Xenopus embryogenesis and the change may be resulted from gene regulation[21]. This is somewhat interesting compared with the situations in mammalian and bird embryos. Now that lamin $\mathrm{L}_{I I}$ is detected in egg extracts and nuclei reassembled in vitro, questions concerning lamin function and expression turn out even more complicated than previously presumed. How do these two polypeptides function and what is their relationship? Though both classified as B-type lamin, do lamin $\mathrm{L}_{I I}$ and $\mathrm{L}_{I I I}$ interact during lamina formation in a manner similar to lamin A and B in mammalian cells? Moreover, the finding of lamin $\mathrm{L}_{I I}$ in egg extracts and nuclei assembled in vitro will help us to explain the obvious contradiction among observations concerning the problem of whether or not the binding of lamin proteins to chromatin surface and lamina formation are required for nuclear envelope assembly in cell-free system[23-25]. In fact when people tried to inactivate endogeneous lamin protein in eggs by immunodepletion or microinjection with antibody, they considered only the presence of lamin $\mathrm{L}_{\text {II I }}$ but neglected lamin $\mathrm{L}_{I I}$ and thus impaired the soundness of their conclusion.

Another phenomenon which interests us is that the somatic chromosomes of $\mathrm{HeLa}$ cells and the sperm chromatin of $X$. laevis can both demonstrate lamina assembly in egg extracts. Previous work has shown that egg extracts are able to induce nuclear assembly around a wide variety of DNA templates[1, 3, 14]. Whether they share the same lamina formation pattern and whether developmentally controlled factors in egg extracts are required for the lamina formation remain unresolved and need further study.

It has been reported that soon after their synthesis in the cytoplasm the lamin proteins, including lamin $\mathrm{L}_{I I}$ and $\mathrm{L}_{I I I}$, dimerize and are transported to the nucleus. A nuclear localization signal and a $\mathrm{CaaX}$ box at the carboxyl terminus are necessary to the latter process[15, 16, 28, 29]. The CaaX motif (C: cysteine, a: an aliphatic amino acid, X: any amino acid) is a substrate for 3 successive post-translational modifications (isoprenylation of the cysteine, proteolytic removal of three carboxyl terminal acids; and carboxyl-methylation of the isoprenylated cysteine). Another kind of modification of lamin proteins is phosphorylation [10]. In all our Westernblotting positive lanes there were two bands at the position of $68 \mathrm{kD}$. Because $\mathrm{mAb}$ $\mathrm{X} 223$ is specific for only lamin $\mathrm{L}_{I I}$, we regard that the doublets in Fig 10 may be resulted from different migrating abilty of two forms of lamin $\mathrm{L}_{I I}$ with different modification. As for the doublets in Fig 8, there may be two possibilities: 1)Each band contains both lamin $\mathrm{L}_{I I}$ and $\mathrm{L}_{I I I}$ and either one of lamin $\mathrm{L}_{I I}$ and $\mathrm{L}_{I I I}$ is of different modification form in the two bands. Because lamin $\mathrm{L}_{I I}$ and $\mathrm{L}_{I I I}$ have similar molecular weight and are both B-type lamin, it is quite possible that after being modified, either in the same manner or not, they have the same mobile activity in our gel system. 2) While both of the two bands contain lamin $\mathrm{L}_{I I}$, only one band contains lamin $\mathrm{L}_{I I}$; in other words, there is only one form of lamin $\mathrm{L}_{I I I}$ which has the same migrating pattern as that of one of the two forms of lamin $\mathrm{L}_{I I}$. Since mAb X167 
is specific for lamin $\mathrm{L}_{I I}$ and two other lamin isoforms but not for lamin $\mathrm{L}_{I I I}$, there is one possibility that the situation in Fig 9 is just like that in Fig 10: each band represents a modification form of lamin $\mathrm{L}_{I I}$. The much slower migrating band in the three figures at the position of approximately $150 \mathrm{kD}$ may be the dimer of lamin proteins, either homodimers of lamin $\mathrm{L}_{I I}$ or $\mathrm{L}_{I I I}$ or heterodimers of lamin $\mathrm{L}_{I I}$ and $\mathrm{L}_{I I I}$.

\section{REFERENCES}

[1] Lokha MJ, Masui Y. Formation in vitro of sperm pronuclei and mitotic chromosomes induced by amphibian ooplasmic components. Science 1983; 220:719-21.

[2] Lokha MJ, Masui Y. Roles of cytosol and cytoplasmic particles in nuclear envelope assembly and sperm pronuclear formation in cell-free preparation from amphibian eggs. J Cell Biol 1984; 98:1222-30.

[3] Newport J. Nuclear reconstitution in vitro: Stages of assembly around protein-free DNA. Cell 1987; 48:205-17.

[4] Newmeyer DD, Lucocq JM, Burglin TR, Derobertis EM. Asssembly in vitro of nuclei and nuclear protein transport: ATP is required for nucleoplasmic accumulation. EMBO 1986; 5:501-10.

[5] Wilson KL, Newport J. A trypsin-sensitive receptor on membrane vesicles is required for nuclear envelope formation in vitro. J Cell Biol 1988; 107:57-68.

[6] Hutchison D J, Cox R, Ford CC. The control of DNA replication in a cell-free extract that recapitulates a basic cell-cycle in vitro. Development 1988; 103:553-66.

[7] Lohka M J, Maller JL. Induction of nuclear envelope breakdown, chromosome condensation, spindle formation in cell-free extracts. J Cell Biol 1985; 101:518-22.

[8] Sawin KE. Mitchison TJ. Mitotic spindle assembly by two different pathways in vitro. J Cell Biol 1991; 112:925-40.

[9] Luscher B, Brizuela L, Beach D, Eiseman RN. A role for the p34 $4^{\text {cdc2 }}$ kinase and phosphotases in the regulation of phosphorylation and disassembly of lamin B2 during the cell cycle. EMBO 1991; 10:865-75.

[10] Peter M, Nakagawa J, Doree M, Labbe JC. Nigg EA. In vitro disassembly of the nuclear lamina and M Phase-specific phosphorylation of lamins by cdc2 kinase. Cell 1990; 61:591-602.

[11] Cai ST, Zhang B, Qing J, Zhang CM, Zhai ZH. Nuclear assembly in vitro using Xenopus egg extracts and Lambda DNA. Chinese Sci Bulletin 1990; 35(16):1261-4.

[12] Zhang CM, Zhang B, Zhai ZH. Chromatin and nucleosome organization and DNA replication of nucleus reassembled in vitro using purified exogenous DNA and Xenopus egg extracts. Sci China(Series B) 1994; 37(6):677-88.

[13] Zhang CM, Qu J, Zhai ZH. Biological activity assays of cell-free reassembled nuclei. Sci China (Series B) 1994; 24(2):165-70.

[14] Zhang CM, Qu J, Zhai ZH. Nuclear reconstitution around purified HeLa chromosomes in a cell-free system from Xenopus eggs. Acta Biol Exp Sin 1994; 27(1):51-9.

[15] Nigg EA. Assembly-disassembly of the nuclear lamina. Cur Opin Cell Biol 1992; 4:105-9.

[16] Mckeon F. Nuclear lamin proteins: domains required for nuclear targeting assembly and cellcycle regulated dynamics. Cur Opin Cell Biol 1991; 3:82-6.

[17] Aebi U, Cohn J, Buhle L, Gerace L. The nuclear lamina is a meshwork of intermediate filaments. Nature 1986; 323:560-4.

[18] Nigg EA. The nuclear envelope. Cur Opin Cell Biol 1989; 1:438-40.

[19] Stick R. cDNA cloning of the developmentally regulated lamin $\mathrm{L}_{I I I}$ of Xenopus laevis. EMBO 1988; 7:3189-97.

[20] Hoger TH, Krohne G, Franke WW. Amino acid sequence and molecular characterization of murine lamin B as deduced from cDNA clones. Eu J Cell Biol 1988; 47:283-90. 


\section{Nuclei assembled in vitro and their lamina components}

[21] Benavente R, Krohne G, Franke W. Cell type-specific expression of nuclear lamina proteins during development of Xenopus laevis. Cell 1985; 41:177-90.

[22] Gerace L, Blobel G. The nuclear envelope is reversibly depolymerized during mitosis. Cell 1980; 19:277-87.

[23] Dabauvalle MC, Loos K, Merkert H, Scheer U. Spontaneous assembly of pore complex-containing membranes ("Annulate Lamellae") in Xenopus egg extracts in the absence of chromatin. J Cell Biol 1991; 112:1073-82.

[24] Newport J, Wilson K, Dunphy W. A lamin-independent pathway for nuclear envelope assembly. J Cell Biol 1990; 111:2247-59.

[25] Burke B, Gerace L. A cell-free system to study reassembly of the nuclear envelope at the end of mitosis. Cell 1986; 44:639-52.

[26] Lourim D, Krohne G. Membrane-associated lamins in Xenopus egg extracts: Identification of two vesicle populations. J Cell Biol 1993; 123:501-12.

[27] Benavente R, Krohne G. Change of karyoskeleton during spermatogenesis of Xenopus: expression of lamin $\mathrm{L}_{\mathrm{I}}$, a nuclear lamina protein specific for the male germ line. Proc Natl Sci 1985; 82:6176-80.

[28] Kitten GT, Nigg EA. The CaaX motif is required for isoprenylation, carboxyl methylation and nuclear membrane association of lamin B2. J Cell Biol 1991; 113:13-23.

[29] Firmbach-Kraft, Stick R. The role of CaaX-dependent modifications in membrane association of Xenopus nuclear lamin B3 during meiosis and the fate of lamin B3 in transfected mitotic cells. J Cell Biol 1993; 123:1661-70.

Received 2-7-1994. Revised 2-11-1994. Accepted 7-11-1994. 